\title{
Isolation and Characterization of Biogenetically Related Highly Oxygenated Nortriterpenoids from Schisandra chinensis
}

Sheng-Xiong Huang, Rong-Tao Li, Jing-Ping Liu, Yang Lu, Ying Chang, Chun Lei, Wei-Lie Xiao, Li-Bin Yang, Qi-Tai Zheng, and Han-Dong Sun

hdsun@mail.kib.ac.cn

State Key Laboratory of Phytochemistry and Plant Resources in West China, Kunming Institute of Botany, Chinese Academy of Sciences, Kunming 650204, P. R.China

Institute of Materia Medica, Chinese Academy of Medical Sciences, Beijing 100050, P. R. China

\section{Supporting Information Available}

Contents:

Page:

1. Experimental Section

2. Proposed biosynthetic pathways leading to pre-schisanartanin (1) and lancifodilactone $\mathrm{G}$

3. $\quad{ }^{1} \mathrm{H}$ NMR data of compounds 1-4 (Table S1) 7

4. Gross semisynthesis route of schisanartane type of triterpenoids 8

5. ${ }^{1} \mathrm{H}$ NMR spectrum of $(R)$-MTPA ester of 5

6. $\quad{ }^{1} \mathrm{H}$ NMR spectrum of $(S)$-MTPA ester of 5

7. COSY spectrum of $(R)$-MTPA esters of 5

8. COSY spectrum of $(S)$-MTPA esters of 5

Note: All citation numbers in the experimental section refer to the bibliography of the manuscript. 


\section{Experimental Section}

General Experimental Procedures: Optical rotations were carried out on a Perkin-Elmer model 241 polarimeter. IR spectrometers were measured in a Bio-Rad FTS-135 spectrometer with KBr pellets. MS were recorded on a VG Auto spec-3000 spectrometer or on a Finnigan MAT 90 instrument. 1D and 2D NMR spectra were taken on a Bruker AM-400 and a Bruker DRX-500 instrument with TMS as internal standard, respectively. Semipreparative HPLC was performed on an Agilent 1100 liquid chromatograph with a Zorbax SB-C $18,9.4 \mathrm{~mm} \times 25 \mathrm{~cm}$ column. Column chromatography were performed either on silica gel (200-300 mesh. Qingdao Marine Chemical Inc., China), Lichroprep RP-18 gel (40-63 $\mu \mathrm{m}$, Merck, Darmstadt, Germany), or MCI gel (75-150 $\mu \mathrm{m}$, Mitsubishi Chemical Corporation, Tokyo, Japan).

Plant Material: The aerial parts of $S$. chinensis were collected in Tonghua prefecture, Jilin Province, People's Republic of China, in September 2005. The sample was identified by Prof. Jun-Lin Yu, and a voucher specimen (KIB 05092106) has been deposited at the State Key Laboratory of Phytochemistry and Plant Resources in West China, Kunming Institute of Botany, Chinese Academy Sciences.

Extraction and Isolation: Air-dried and powdered aerial parts of S. chinensis (4.5 kg) were extracted with acetone $(5 \mathrm{~L} \times 4$, each 2 days $)$ at room temperature. After evaporating the solvents in vacuo at $45{ }^{\circ} \mathrm{C}$, a residue $(207 \mathrm{~g})$ was obtained. This residue was dissolved in $\mathrm{H}_{2} \mathrm{O}(3.0 \mathrm{~L})$ and then extracted successively with petroleum ether $\left(60-90{ }^{\circ} \mathrm{C}, 1.5 \mathrm{~L} \times 2\right)$ and EtOAc $(2 \mathrm{~L} \times 3)$. The EtOAc extract $(102 \mathrm{~g})$ was chromatographed on MCI-gel CHP 20P (90\% MeOH- $\left.\mathrm{H}_{2} \mathrm{O}, 100 \% \mathrm{MeOH}\right)$. The $90 \%$ $\mathrm{MeOH}$ fraction (81 g) was subjected to column chromatography over silica gel (200-300 mesh) and eluted with $\mathrm{CHCl}_{3}-\mathrm{Me}_{2} \mathrm{CO}$ (from 1:0 to 0:1) to give fractions A-E. Fraction D (8.2 g) was chromatographed on RP-18 eluted with a $\mathrm{MeOH}-\mathrm{H}_{2} \mathrm{O}$ (30\%-80\%) gradient system to afford four main subfractions, D1-D5. Lancifodilactone C (6) (65.4 mg) was obtained from subfractions D1 (0.85 g) by recrystallization from $\mathrm{CH}_{3} \mathrm{OH}$. The remainder of D1 (0.80 g) was subjected to silica gel column chromatography, eluted with petroleum ether-isopropyl alcohol (5:1), to give schindilactone C (4) (7.5 mg) and henridilactone D (7) (42.5 mg). Subfraction D2 
(0.63 g) was chromatographed on silica gel using petroleum ether-isopropyl alcohol (6:1) as solvent, and finally purified by semipreparative HPLC using $3 \mathrm{~mL} / \mathrm{min}$ $\mathrm{MeOH}-\mathrm{H}_{2} \mathrm{O}(45: 55)$ as the mobile phase to yield compounds 2 (13.0 mg) and 3 (7.4 mg). Micrandilactone B (5) (83.6 mg) was obtained from subfraction D3 (2.80 g) by recrystallization from $\mathrm{CH}_{3} \mathrm{OH}$. The remainder of subfraction D3 was subjected to column chromatography over silica gel and eluted with $\mathrm{CHCl}_{3}-\mathrm{Me}_{2} \mathrm{CO}(8: 1)$ to give $\mathbf{1}$ (59.2 mg).

Pre-schisanartanin (1): colorless crystals; mp $178-179{ }^{\circ} \mathrm{C} ;[\alpha]_{\mathrm{D}}^{19}=80.1^{\circ}(c 0.50$ in $\left.\mathrm{CH}_{3} \mathrm{OH}\right)$; IR(KBr) $v_{\max }=3441,2934,2871,1764,1739,1638,1456,1373,1238,1069$, 1035, $924 \mathrm{~cm}^{-1} ;{ }^{1} \mathrm{H}$ NMR see Table S1; ESI-MS: $m / z 611[\mathrm{M}+\mathrm{Na}]^{+}$; HRESIMS: $m / z$ $611.2462[\mathrm{M}+\mathrm{Na}]^{+}$, calcd for $\mathrm{C}_{31} \mathrm{H}_{40} \mathrm{O}_{11} \mathrm{Na}: m / z 611.2468$.

Schindilactone A (2): colorless crystals; mp 254-255 ${ }^{\circ} \mathrm{C} ;[\alpha]_{\mathrm{D}}^{19}=101.5^{\circ}(c 0.80$ in $\left.\mathrm{CH}_{3} \mathrm{OH}\right)$; IR(KBr) $v_{\max }=3441,2963,2929,1787,1739,1659,1460,1377,1343,1111$, $1010 \mathrm{~cm}^{-1}$; ${ }^{1} \mathrm{H}$ NMR see Table S1; FABMS: $m / z 543[\mathrm{M}+\mathrm{H}]^{+}$; HRESIMS: $\mathrm{m} / z$ $565.2043[\mathrm{M}+\mathrm{Na}]^{+}$, calcd for $\mathrm{C}_{29} \mathrm{H}_{34} \mathrm{O}_{10} \mathrm{Na}: m / z$ 565.2049.

Schindilactone B (3): white solid; $\mathrm{mp} 261-262{ }^{\circ} \mathrm{C}$; $[\alpha]_{\mathrm{D}}^{19}=55.6^{\circ}\left(c 0.10\right.$ in $\left.\mathrm{CH}_{3} \mathrm{OH}\right)$; $\operatorname{IR}(\mathrm{KBr}) \nu_{\max }=3443,2974,2933,1776,1734,1666,1457,1208,1153,1109,1008$ $\mathrm{cm}^{-1} ;{ }^{1} \mathrm{H}$ NMR see Table S1; FABMS: $\mathrm{m} / z, 543[\mathrm{M}+\mathrm{H}]^{+}$; HRESIMS: $\mathrm{m} / z 565.2042[\mathrm{M}$ $+\mathrm{Na}]^{+}$, calcd for $\mathrm{C}_{29} \mathrm{H}_{34} \mathrm{O}_{10} \mathrm{Na}: m / z$ 565.2049.

Schindilactone C (4): white solid; $\operatorname{mp} 221-222^{\circ} \mathrm{C}$; $[\alpha]_{\mathrm{D}}^{19}=89.4^{\circ}\left(c 0.15\right.$ in $\left.\mathrm{CH}_{3} \mathrm{OH}\right)$; $\operatorname{IR}(\mathrm{KBr}) v_{\max }=3442,2973,2934,1755,1639,1459,1382,1253,1202,1100,1017$ $\mathrm{cm}^{-1} ;{ }^{1} \mathrm{H}$ NMR see Table S1; FABMS: $m / z, 561[\mathrm{M}+\mathrm{H}]^{+}$; HRESIMS: $m / z 583.2164[\mathrm{M}$ $+\mathrm{Na}]^{+}$, calcd for $\mathrm{C}_{29} \mathrm{H}_{36} \mathrm{O}_{11} \mathrm{Na}: m / z 583.2155$.

X-ray Crystallographic Analysis of 1: $\mathrm{C}_{31} \mathrm{H}_{40} \mathrm{O}_{11}, M=588.63$, monoclinic, space group $P 2_{1}, a=11.132$ (1), $b=12.407$ (1), $c=11.655$ (1) $\AA, V=1533.8$ (2) $\AA^{3}, Z=2, \beta$ $=107.67(1)^{\mathrm{o}}$, Dcalc $=1.275 \mathrm{~g} \mathrm{~cm}^{-3}$, crystal dimentions $0.20 \times 0.40 \times 0.60 \mathrm{~mm}$. The total number of independent reflections measured was 3390, of which 3294 were observed $(|F| 2 \geq 8 \sigma|F| 2)$. The final indices were $R_{\mathrm{f}}=0.0656, R_{\mathrm{w}}=0.1814(w=1 / \sigma|F| 2)$, $\mathrm{S}=1.071$ (Figure $\mathrm{S} 1$ ). 

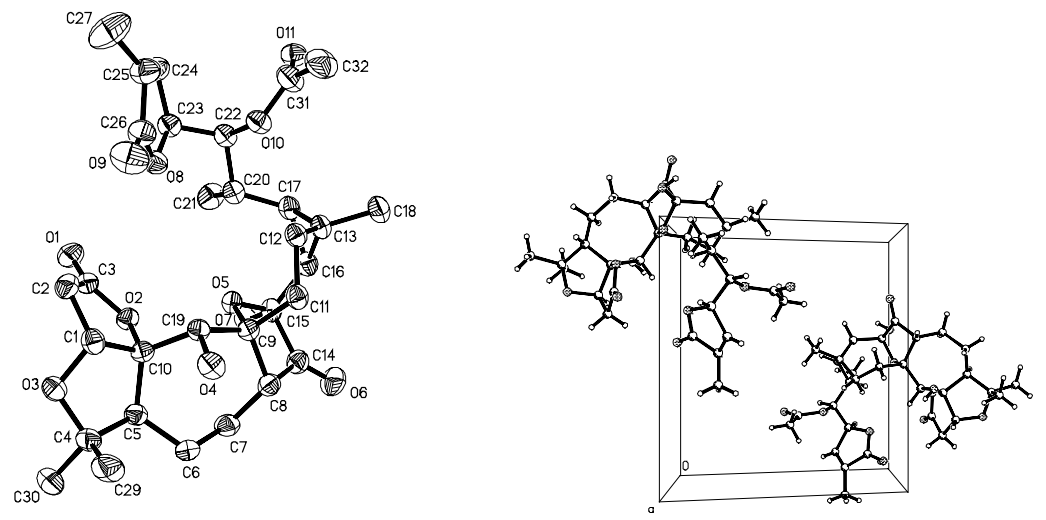

Figure S1. X-ray structure of 1

X-ray Crystallographic Analysis of 2: Dimension: $0.10 \times 0.30 \times 0.50 \mathrm{~mm}$. $\left(\mathrm{C}_{29} \mathrm{H}_{34} \mathrm{O}_{10}\right), \mathrm{M}=534.65$, triclinic, space group, $\mathrm{P} 2{ }_{1}, \mathrm{a}=8.214(1), \mathrm{b}=15.608(1), \mathrm{c}=$ 11.522 (1) $\AA, \beta=90.98(1)^{\circ}, Y=89.66(1)^{\circ}, V=1476.9(7) \AA^{3}, Z=2, d=1.301 \mathrm{~g} \mathrm{~cm}^{-3}$. The total number of independent reflections measured was 3054, of which 1896 were observed. $R_{\mathrm{f}}=0.0429, R_{\mathrm{w}}=0.1266(\mathrm{w}=1 / \sigma|\mathrm{F}| 2), \mathrm{S}=1.031$ (Figure $\left.\mathrm{S} 2\right)$.
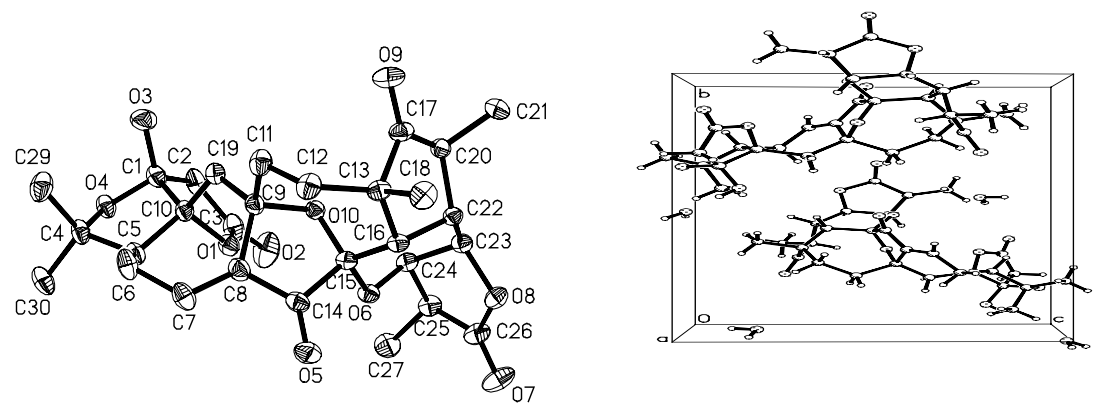

Figure S2. X-ray structure of 2

All measurements of both crystal structures were made on a MAC DIP-2030K diffractometer with graphite-monochromated Mo $\mathrm{K} \alpha$ radiation. The data were collected by using the $\omega-2 \theta$ scan technique to a maximum $2 \theta$ value of $50.0^{\circ}$. The crystal structures were solved by direct the method SHELX-86 (Sheldrick, G. M. University of Gottingen, Federal Republic of Germany, 1985), expanded using difference Fourier techniques, refined by the program and method NOMCSDP (Y. Lu, B. M. Wu, Chin. Chem. Lett. 1992, 3, 637-640), and full-matrix least-squares 
calculations. The non-hydrogen atoms were fined anisotropically and hydrogen atoms were included at their calculated positions. Crystallagraphic data for $\mathbf{1}$ and $\mathbf{2}$ have been deposited in the Cambridge Crystallographic Data Centre (deposition number: CCDC 636567 and 636568). Copies of this data can be obtained, free of charge, on application to the CCDC via www.ccdc.com.ac.uk/conts/retrieving.html (or 12 Union Road, Cambridge CB2 1EZ, UK, fax: + 441223 336033, e-mail: deposit@ccdc.cam.ac.uk). Absolute Configuration of 5: Micrandilactone B (5) was derivatized with $(R)$ - and $(S)$-MTPACl. To $3.0 \mathrm{mg}$ of micrandilactone B was added $0.5 \mathrm{~mL}$ of anhydrous DCM under an atmosphere of nitrogen at room temperature. Following a 30 min incubation period with vigorous stirring, $10 \mu \mathrm{L}$ of pyridine was added, followed 5 min later by the addition of 1 crystal of dimethylaminopyridine. The reaction components were stirred for $60 \mathrm{~min}$, and then $5 \mu \mathrm{L}$ of $(R)$ - or $(S)$-MTPACl was added. The reaction was incubated for another $60 \mathrm{~min}$, at the end of which $300 \mu \mathrm{L}$ of $\mathrm{C}_{5} \mathrm{H}_{5} \mathrm{~N}$ was added. After an hour stirring, the reaction was dried in vacuo and subsequently purified by RP-18 column eluted with $\mathrm{MeOH} / \mathrm{H}_{2} \mathrm{O}(45 \%)$. Comparison of the ${ }^{1} \mathrm{H}$ NMR spectra of $\mathbf{5 a}$ and 5b clearly indicated its $S$ configuration of C-22.

Cytotoxicity and HIV-1 Inhibition Assay: The anti-HIV activities and cytotoxicities of 1-7 were tested by microtiter syncytium formation infectivity assay, using the method previously described, with AZT as a positive control. The assays include cytotoxicity in C8166 and MT-4 cells, inhibition of syncytium formation in HIV-1IIIB infected C8166 cells, and effect in protecting HIV-1IIIB infected MT-4 host cells from dying.

Scheme S1. Proposed biosynthetic pathway leading to pre-schisanartanin (1). 


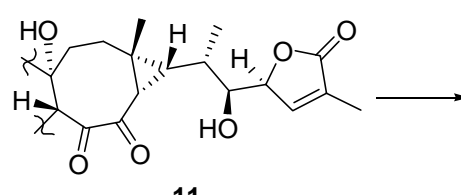

11

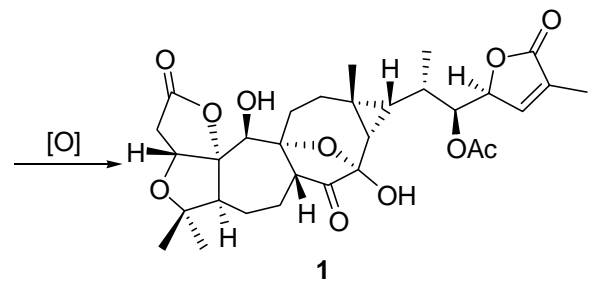

Scheme S2. Proposed biosynthetic pathway leading to lancifodilactone G.
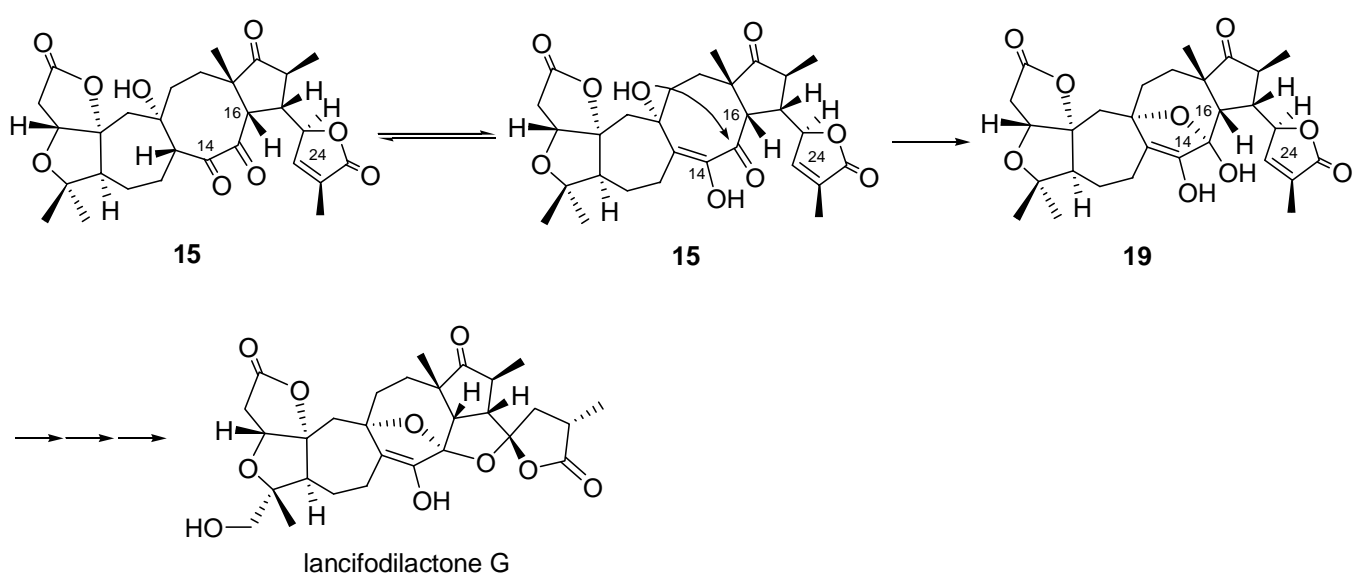
Table S1. ${ }^{1} \mathrm{H}$ NMR data of compounds $1-4$ (pyridine- $d_{5}, 400 \mathrm{MHz}, \delta$ in ppm, $J$ in $\left.\mathrm{Hz}\right)^{a}$

\begin{tabular}{|c|c|c|c|c|c|c|c|c|}
\hline position & 1 & & 2 & & 3 & & 4 & \\
\hline 1 & 5.02 & $\mathrm{~d}, J=5.2$ & & & & & & \\
\hline $2 \alpha$ & 2.72 & $\mathrm{~d}, J=18.1$ & 3.20 & $\mathrm{~s}$ & 3.17 & $\mathrm{~s}$ & 3.16 & $\mathrm{~s}$ \\
\hline $2 \beta$ & 3.32 & $\mathrm{dd}, J=18.1,5.2$ & 3.21 & $\mathrm{~s}$ & 3.18 & $\mathrm{~s}$ & 3.17 & $\mathrm{~s}$ \\
\hline 5 & 2.18 & overlapped & 2.29 & overlapped & 2.30 & overlapped & 2.46 & $\mathrm{dd}, J=12.9,4.0$ \\
\hline $6 \alpha$ & 2.17 & overlapped & 2.29 & overlapped & 2.31 & overlapped & 2.26 & $\mathrm{~m}$ \\
\hline $6 \beta$ & 1.37 & overlapped & 2.14 & overlapped & 2.18 & $\mathrm{~m}$ & 2.14 & $\mathrm{~m}$ \\
\hline $7 \alpha$ & 2.28 & $\mathrm{~m}$ & 7.06 & t like, $J=3.8$ & 7.05 & $\mathrm{t}$ like, $J=3.8$ & 4.74 & $\mathrm{~m}$ \\
\hline $7 \beta$ & 2.02 & $\mathrm{~m}$ & & & & & & \\
\hline 8 & 2.85 & $\mathrm{dd}, J=7.0,4.5$ & & & & & 2.88 & $\mathrm{~d}, J=10.0$ \\
\hline $11 \alpha$ & 1.47 & $\mathrm{~m}$ & 1.69 & $\mathrm{~m}$ & 1.68 & $\mathrm{~m}$ & $1.70-1$ & $1.85 \mathrm{~m}$ \\
\hline $11 \beta$ & 2.71 & $\mathrm{~m}$ & 2.14 & overlapped & 2.34 & overlapped & $1.70-1$ & $1.85 \mathrm{~m}$ \\
\hline $12 \alpha$ & 1.38 & overlapped & 1.45 & $\mathrm{~m}$ & 1.45 & $\mathrm{~m}$ & 1.78 & $\mathrm{~m}$ \\
\hline $12 \beta$ & 2.49 & $\mathrm{~m}$ & 1.87 & $\mathrm{~m}$ & 1.84 & $\mathrm{~m}$ & 1.98 & $\mathrm{~m}$ \\
\hline \multicolumn{9}{|l|}{14} \\
\hline \multicolumn{9}{|l|}{$16 \alpha$} \\
\hline $16 \beta$ & 1.41 & $\mathrm{~d}, J=7.0$ & 2.75 & $\mathrm{~d}, J=6.5$ & 2.76 & overlapped & 3.24 & $\mathrm{~d}, J=8.1$ \\
\hline 17 & 0.84 & $\mathrm{t}$ like, $J=7.0$ & & & & & & \\
\hline 18 & 0.95 & $\mathrm{~s}$ & 0.94 & $\mathrm{~s}$ & 0.96 & $\mathrm{~s}$ & 1.06 & $\mathrm{~s}$ \\
\hline $19 \alpha$ & 4.20 & $\mathrm{~d}, J=7.3$ & 2.58 & $\mathrm{ABd}, J=16.4$ & 2.63 & $\mathrm{ABd}, J=16.4$ & 2.64 & $\mathrm{ABd}, J=16.5$ \\
\hline $19 \beta$ & & & 2.75 & $\mathrm{ABd}, J=16.4$ & 2.77 & $\mathrm{ABd}, J=16.4$ & 2.88 & $\mathrm{ABd}, J=16.5$ \\
\hline 20 & 3.67 & $\mathrm{~m}$ & 2.61 & $\mathrm{~m}$ & 3.39 & $\mathrm{~m}$ & 2.75 & $\mathrm{~m}$ \\
\hline 21 & 1.70 & $\mathrm{~d}, J=6.4$ & 1.25 & $\mathrm{~d}, J=6.5$ & 1.33 & $\mathrm{~d}, J=7.0$ & 1.03 & $\mathrm{~d}, J=7.0$ \\
\hline 22 & 5.11 & br d, $J=8.1$ & 2.84 & $\mathrm{~m}$ & 2.81 & $\mathrm{~m}$ & 3.07 & $\mathrm{~m}$ \\
\hline 23 & 5.36 & brs & 4.51 & brs & 4.66 & brs & 6.39 & $\mathrm{~d}, J=1.8$ \\
\hline 24 & 7.09 & brs & 4.75 & $\mathrm{dd}, J=4.0,2.0$ & 4.63 & $\mathrm{dd}, J=4.0,2.0$ & 8.62 & brs \\
\hline 25 & & & 3.09 & $\mathrm{~m}$ & 3.14 & $\mathrm{~m}$ & & \\
\hline 27 & 1.81 & br s & 1.64 & $\mathrm{~d}, J=7.2$ & 1.61 & $\mathrm{~d}, J=7.2$ & 2.32 & brs \\
\hline 29 & 1.32 & s & 1.31 & $\mathrm{~s}$ & 1.33 & $\mathrm{~s}$ & 1.39 & $\mathrm{~s}$ \\
\hline 30 & 1.17 & $\mathrm{~s}$ & 1.26 & $\mathrm{~s}$ & 1.26 & $\mathrm{~s}$ & 1.20 & $\mathrm{~s}$ \\
\hline
\end{tabular}

${ }^{a}$ The assignments were based on ${ }^{1} \mathrm{H}-{ }^{1} \mathrm{H}$ COSY, HSQC, and HMBC experiments. 
Gross semisynthesis route of schisanartane type of triterpenoids.
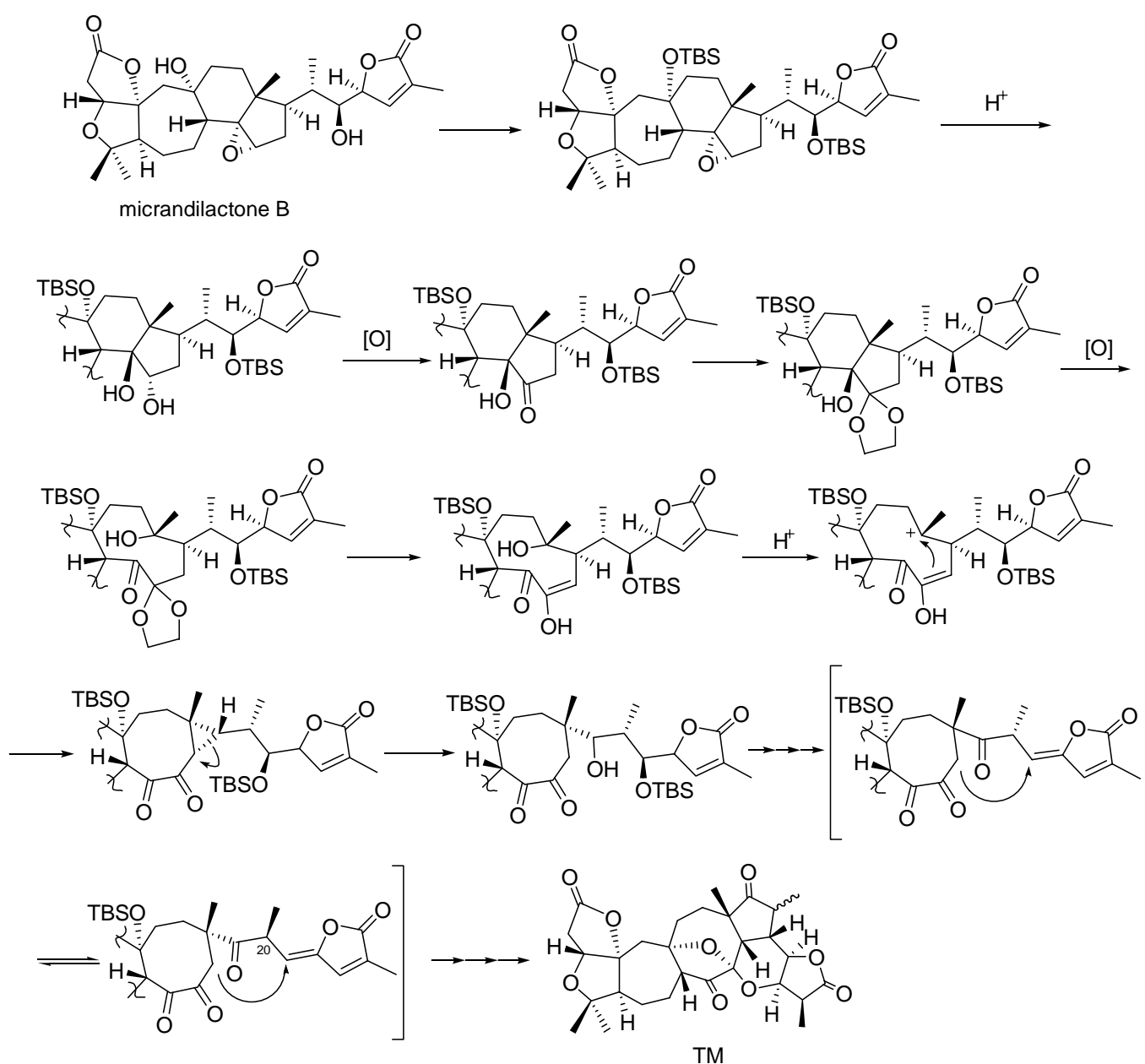


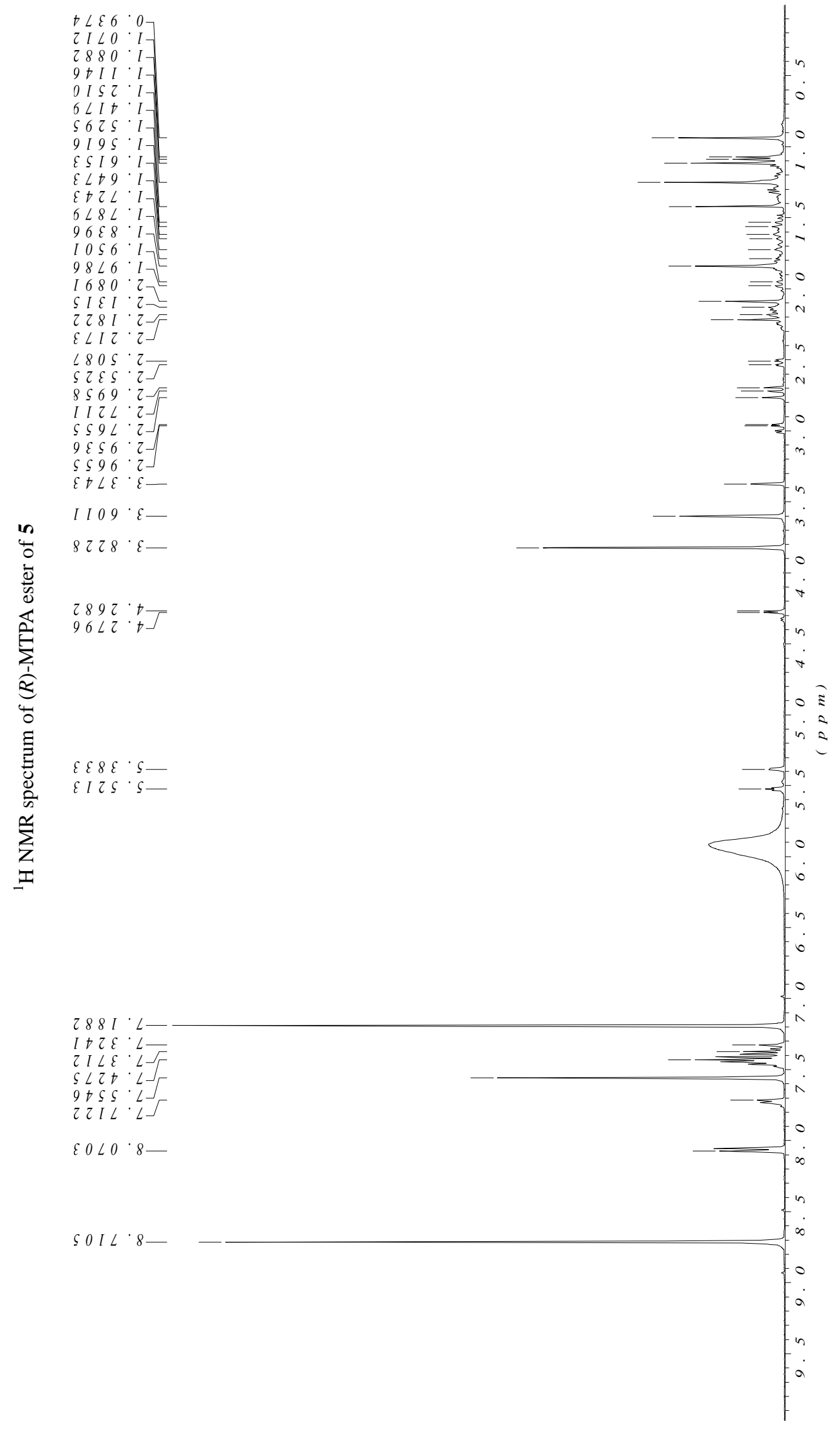

$a$ 


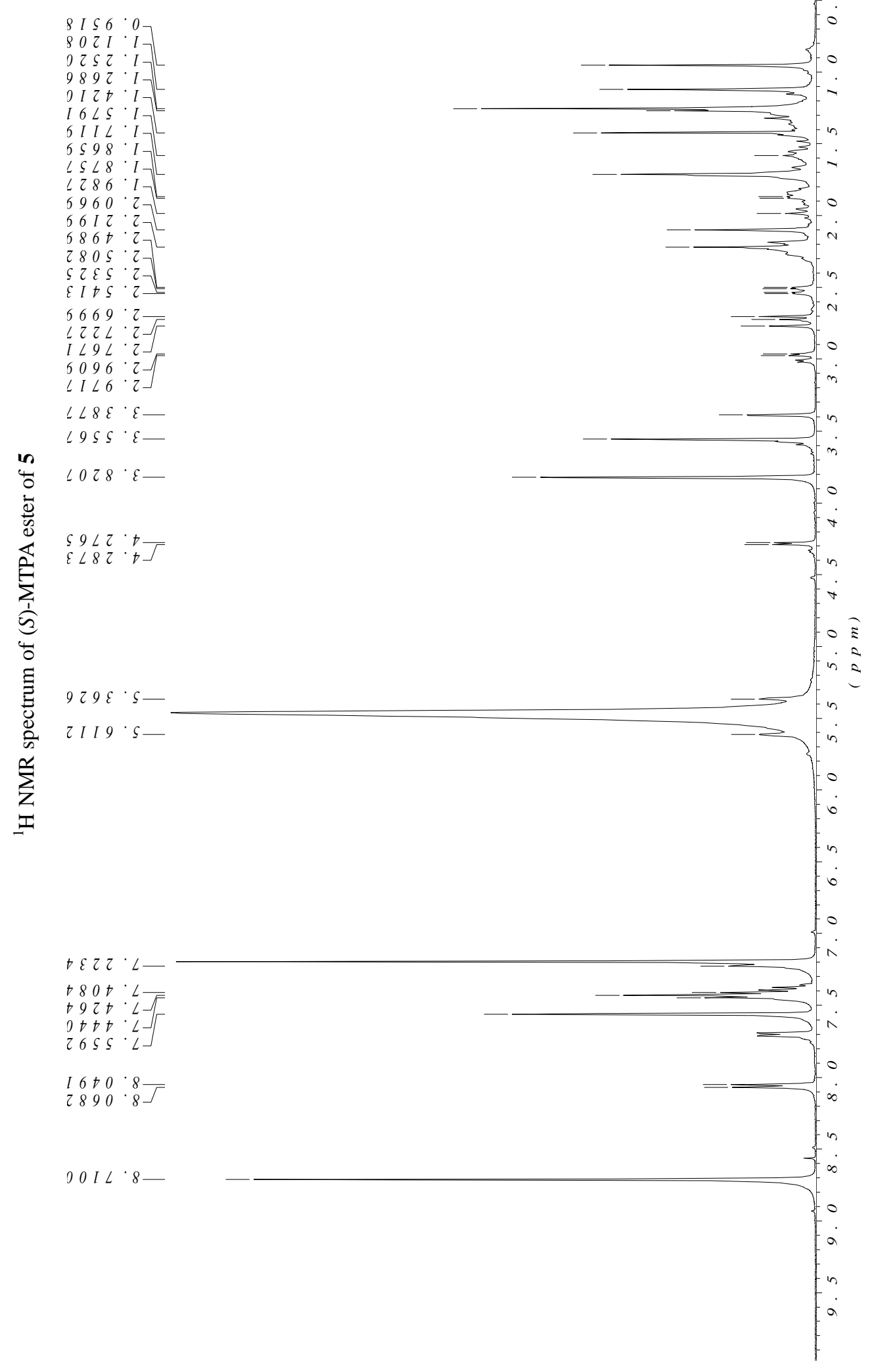

인 


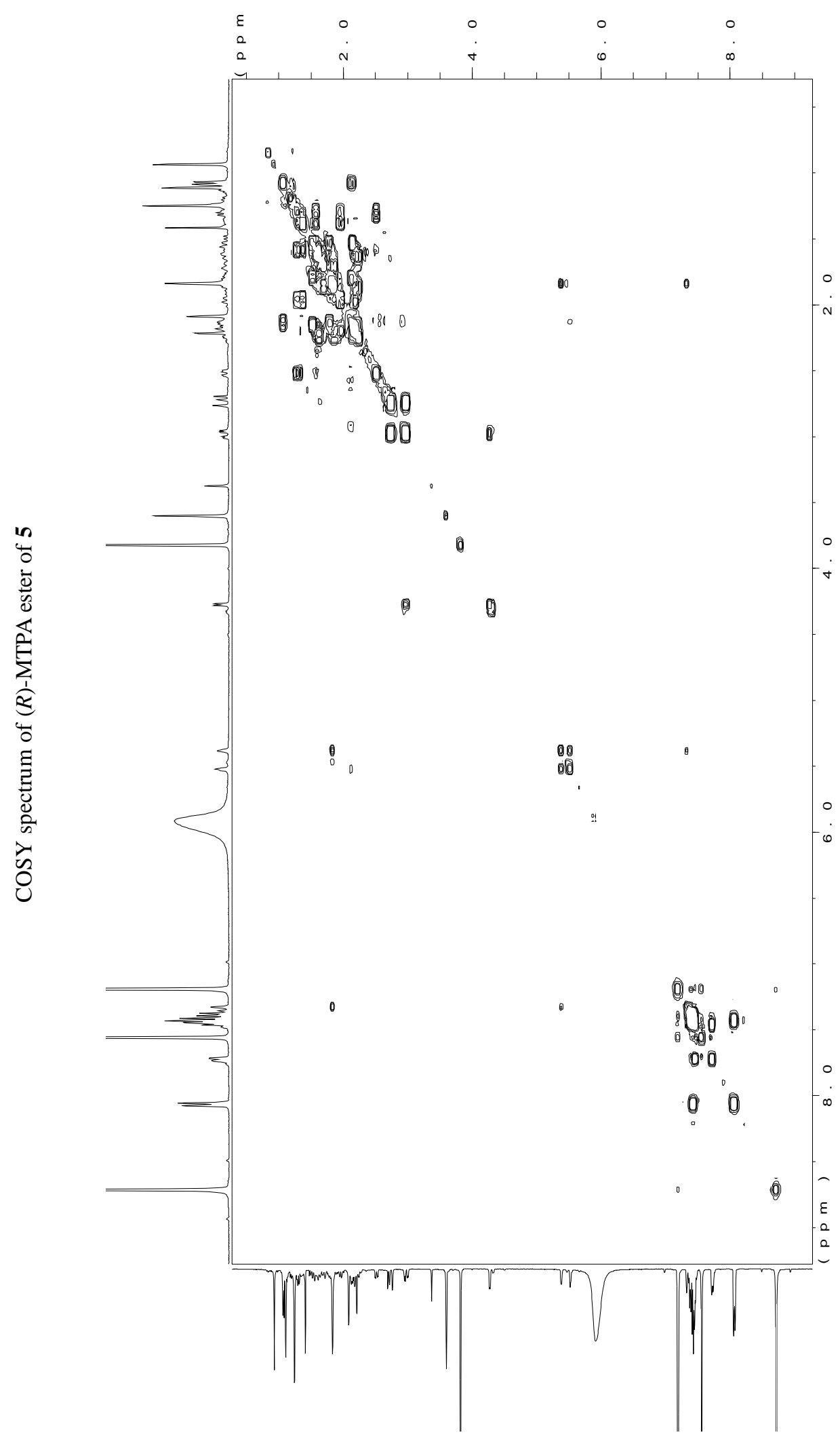




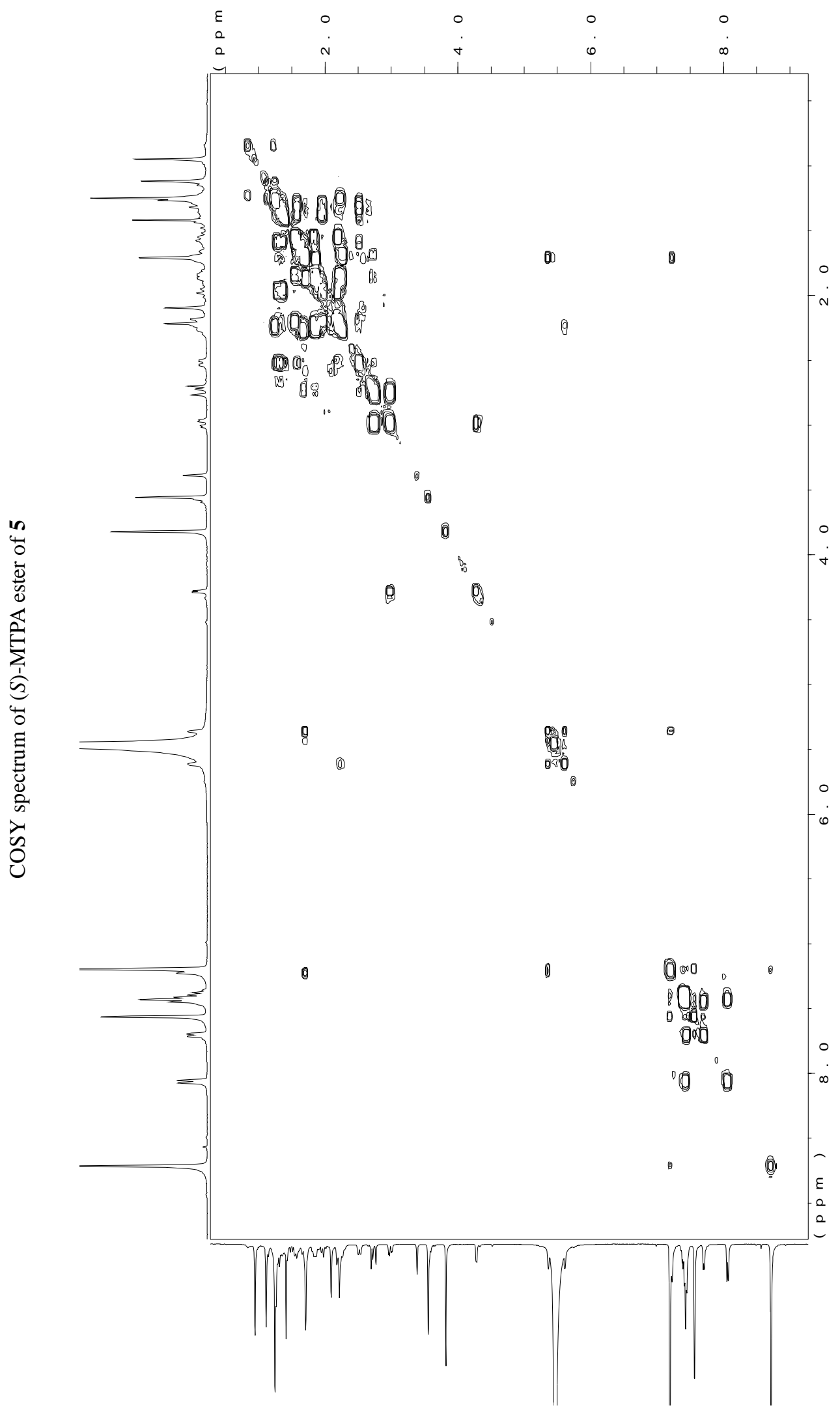

$\simeq$ 